Check for updates

Cite this: RSC Adv., 2019, 9, 31797

\title{
Observation of giant spin-orbit interaction in graphene and heavy metal heterostructures $\dagger$
}

\author{
Amir Muhammad Afzal, Kuen Hong Min, Byung Min Ko and Jonghwa Eom (DD*
}

\begin{abstract}
Graphene is a promising material demonstrating some interesting phenomena such as the spin Hall effect, bipolar transistor effect, and non-trivial topological states. However, graphene has an intrinsically small spin-orbit interaction (SOI), making it difficult to apply in spintronic devices. The electronic band structure of graphene makes it possible to develop a systematic method to enhance SOI extrinsically. In this study, we designed a graphene field-effect transistor with a $\mathrm{Pb}$ layer intercalated between graphene (Gr) and Au layers and studied the effect on the strength of the SOI. The SOI in our system was significantly increased to $80 \mathrm{meV}$, which led to a giant non-local signal $(\sim 180 \Omega)$ at room temperature due to the spin Hall effect. Further, we extract key parameters of spin transport from the length and width dependence of non-local measurement. To support these findings, we also measured the temperature and gate-dependent weak localization (WL) effect. We obtained the magnitude of the SOI and spin relaxation time of $\mathrm{Gr}$ via quantitative analysis of WL. The SOI magnitudes estimated from the non-local signal and the WL effect are close in value. The enhancement of the SOI of $\mathrm{Gr}$ at room temperature is a potential simple manipulation method to explore the use of this material for spin-based applications.
\end{abstract}

Received 2nd September 2019

Accepted 1st October 2019

DOI: $10.1039 / c 9 r a 06961 \mathrm{e}$

rsc.li/rsc-advances ambient conditions because these atoms can be easily unglued at moderate temperature.

Zhang et al. ${ }^{\mathbf{1 4}}$ and Weeks et al. ${ }^{15}$ proposed a similar approach to enhance SOI in Gr. They recommended depositing heavy metals onto the hollow centre of the honeycomb lattice of the carbon sheet that modified the SOI in Gr and opened a bandgap at the Dirac point. Similarly, Ir and W $5 \mathrm{~d}$ atoms can intercede in a quantum anomalous Hall state. ${ }^{\mathbf{1 4}}$ Alternatively, d-block metals such as $\mathrm{Au}, \mathrm{Ti}$, and In have strong SOIs and can open a bandgap at the Dirac point in $\mathrm{Gr}$; they can also introduce the quantum spin Hall effect in the Gr sheet. ${ }^{15} 4 \mathrm{~d}$ and $5 \mathrm{~d}$ heavy transition metals demonstrate intriguing physical properties necessary for spintronics devices, especially when interlinked with a magnetic materials. ${ }^{16,17}$ Despite these promising applications, one genuine problem that remains is that the surface adsorption induces additional electronics scattering. The coherence of the Dirac fermions can be suppressed by such electronic scattering. ${ }^{\mathbf{1 4 , 1 5 , 1 8}}$ Recently, a van der Waals (vdW) heterostructure assembled by vertically staking $\mathrm{Gr}$ and twodimensional materials resulted in a compact system with possibility to manipulate and modulate the spin-based fieldeffect transport properties. The hybridization of transition metal dichalcogenides (TMDs) or Gr with magnetic materials can strongly modulate the electronic structure via the proximity effect. ${ }^{19-21}$ However, suggestions for how to begin SOI enhancement in Gr and spin absorption in twodimensional TMDs are still elusive or remain
Department of Physics \& Astronomy, Graphene Research Institute-Texas Photonics Center International Research Center (GRI-TPC IRC), Sejong University, Seoul 05006, Korea.E-mail: eom@sejong.ac.kr

$\dagger$ Electronic supplementary information (ESI) available. See DOI: 10.1039/c9ra06961e

Graphene (Gr), with its honey-comb structure of carbon atoms, has enormous potential for used in spintronics, electronics, become a strong contender for next-generation spin-based devices owing to its high mobility, weak hyperfine coupling, emperature. ${ }^{4-6}$ However, its lack of an intrinsic spinmake it difficult to apply $\mathrm{Gr}$ to spintronic devices. ${ }^{7}$ The SOI in $\mathrm{Gr}$ has attracted much theoretical attention to date but is less investigated experimentally. ${ }^{8}$

manipulating its SOI due to its surface sensitivity. ${ }^{9}$ Different ocedures have been proposed to improve the SOI in Gr. by creating a curve in flat $\mathrm{Gr}$ and by carbon nanotubes. ${ }^{\mathbf{1 0 , 1 1}}$ meV of hydrogen and 5.1-9.1 meV of fluorine. Unfortunately, these chemisorbed light atoms make devices unstable in 
controversial. ${ }^{22-24}$ These approaches are responsible for disorders in the charge transport quality and have a number of limitations for spin transport, making it problematic for use in controlling the SOI in Gr. Moreover, an elusive challenge in this field is the realization of enhanced SOI and induced topological nontrivial gaps inside the Dirac point. In this work, we made a $\mathrm{Gr} / \mathrm{Pb} / \mathrm{Au}$ heterostructure field effect transistor (FET). For the first time, we used $\mathrm{Pb}$ as an intercalated layer between the graphene and Au and measured SOI in the heterostructure device in local and non-local measurement configurations. Intercalation of the $\mathrm{Pb}$ thin layer between $\mathrm{Au}$ and Gr leads to significant modification of the electronic structure and SOI of the latter. We observed a giant spin Hall effect $\left(R_{\mathrm{NL}} \sim 180 \Omega\right)$ in the device without (a)
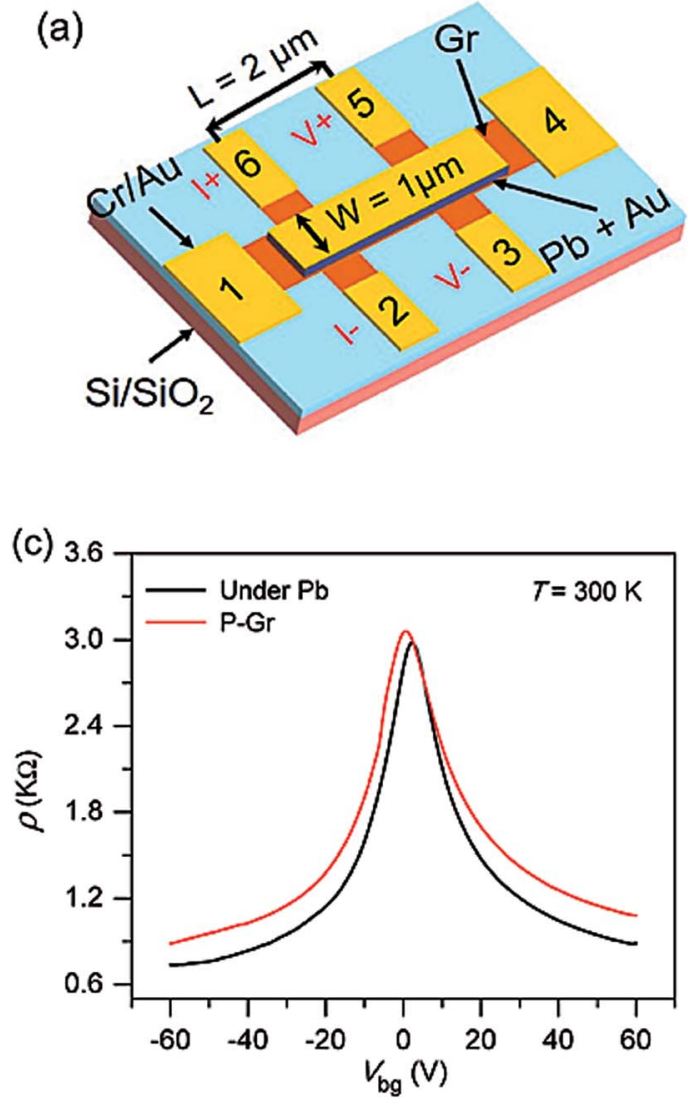

(b)

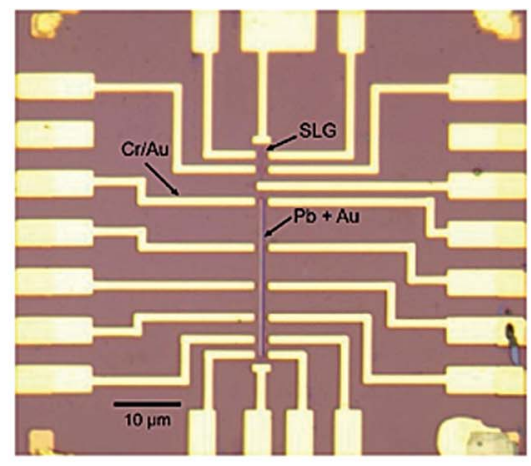

(d) 1.4

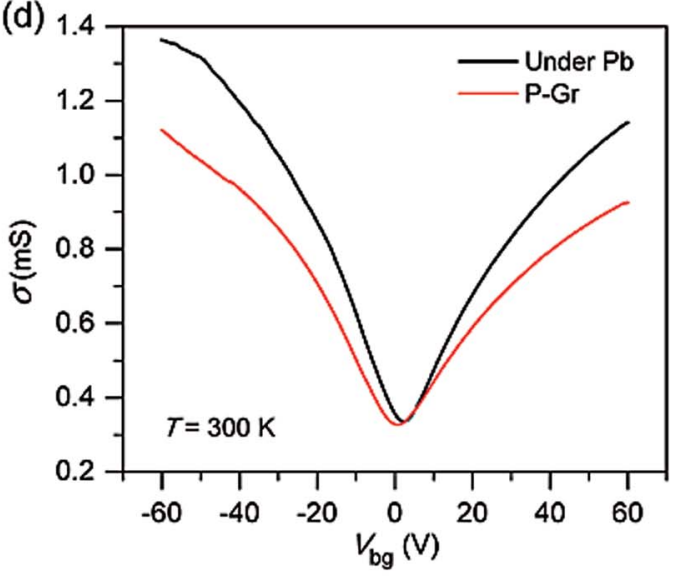

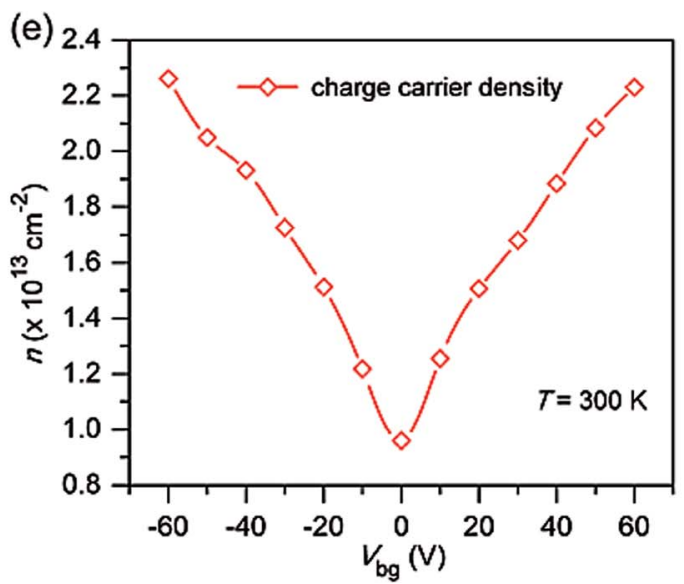

Fig. 1 (a) Schematic of graphene (Gr) device for local and non-local configuration with lead (Pb) and without Pb. In non-local measurement, we applied a current between 6 and 2 and perceived the voltage drop between 5 and 3. For local measurement, we applied a current between electrodes 1 and 4 and detected the voltage drop between 2 and 3 . In each case, the length and width of the channel is $L=2 \mu \mathrm{m}$ and $W=1 \mu \mathrm{m}$, respectively. (b) Optical image of a completed device with multiple Hall bars on pristine graphene (P-Gr) and graphene with $\mathrm{Pb}+\mathrm{Au}$. (c) Resistivity of $\mathrm{P}-\mathrm{Gr}$ and covered graphene with $\mathrm{Pb}+\mathrm{Au}$ as a function of back-gate voltages at room temperature. (d) Conductivity of $\mathrm{P}-\mathrm{Gr}$ and covered graphene as a function of back-gate voltages at room temperature. (e) Charge carrier density as a function of back-gate voltages. 
ferromagnetic electrodes. To further quantitatively examine the improvement in the magnitude of SOI of Gr, we fit our magneto-conductivity data to an equation from the theory of weak localization (WL). The SOI values calculated for $\mathrm{Gr}$ through non-local configuration and WL analysis are similar, supporting the internal consistency of experimental results in this study. The effective improvement of SOI in the Gr-based system opens a new gateway to explore new areas of the spin FET phenomenon.

\section{Characterization of the $\mathrm{SLG} / \mathrm{Pb}+\mathrm{Au}$ heterostructure device}

Fig. 1a is a schematic of the $\mathrm{Gr} / \mathrm{Pb} / \mathrm{Au}$ heterostructure device and Fig. $1 \mathrm{~b}$ is an optical image of the final device in one of its two basic Hall bar configurations. In one of these configurations, $\mathrm{Gr}$ is underneath the $\mathrm{Pb}$ and $\mathrm{Au}$; in the other, the $\mathrm{Gr}$ does not have any top layers. The Raman spectra of graphene on $\mathrm{SiO}_{2}$ are shown in Fig. S1c, $\dagger$ in which the $\mathrm{G}$ and $2 \mathrm{D}$ peaks appear around $1580 \mathrm{~cm}^{-1}$ and $2700 \mathrm{~cm}^{-1}$. The ratio of intensities of $\mathrm{G}$ and $2 \mathrm{D}$ peaks $\left(I_{2 \mathrm{D}} / I_{\mathrm{G}}\right)$ is $\sim 2.6$, which is related to a previously reported value of single layer $\mathrm{Gr}$ (SLG). ${ }^{25,26}$ Atomic force microscopy (AFM) was used to confirm the thickness. Fig. $\mathrm{S} 2 \mathrm{~b} \dagger$ shows the height profile of Gr on $\mathrm{SiO}_{2}$, with the thickness of SLG being $\sim 0.337 \mathrm{~nm}$.

First, the charge transport was characterized. Fig. 1c and d show resistivity and conductivity as a function of back-gate voltage. The back-gate voltage was swept from $-60 \mathrm{~V}$ to $+60 \mathrm{~V}$ and resistivity and conductivity were measured. In cases of pristine Gr, the charge neutrality point (CNT) (i.e., the Dirac point) lies nearly at zero back-gate voltage. When $\mathrm{Pb}$ is deposited, the Dirac point shifts in a positive direction. The mobility of Gr can be calculated by $\mu=\left(1 / C_{\mathrm{g}}\right)(\partial \sigma / \partial V)$, where $C_{\mathrm{g}}$ is the gate capacitance and $\sigma$ is conductivity. The calculated mobilities are $25000 \mathrm{~cm}^{2} \mathrm{~V}^{-1} \mathrm{~s}^{-1}$ and $29100 \mathrm{~cm}^{2} \mathrm{~V}^{-1}$ $\mathrm{S}^{-1}$ in pristine graphene and graphene with $\mathrm{Pb}+\mathrm{Au}$, respectively. ${ }^{27-30}$ Furthermore, we measured the charge carrier density as a function of back-gate voltage at room temperature, as shown in Fig. 1e. For the charge carrier density, we measured the Hall measurement at the different back-gates and calculated charge carrier density using $n=1$ / $\left(e R_{x y} / B\right)$, where $R_{x y}$ is the Hall resistance at magnetic field $B$ and $e$ is the charge of an electron.
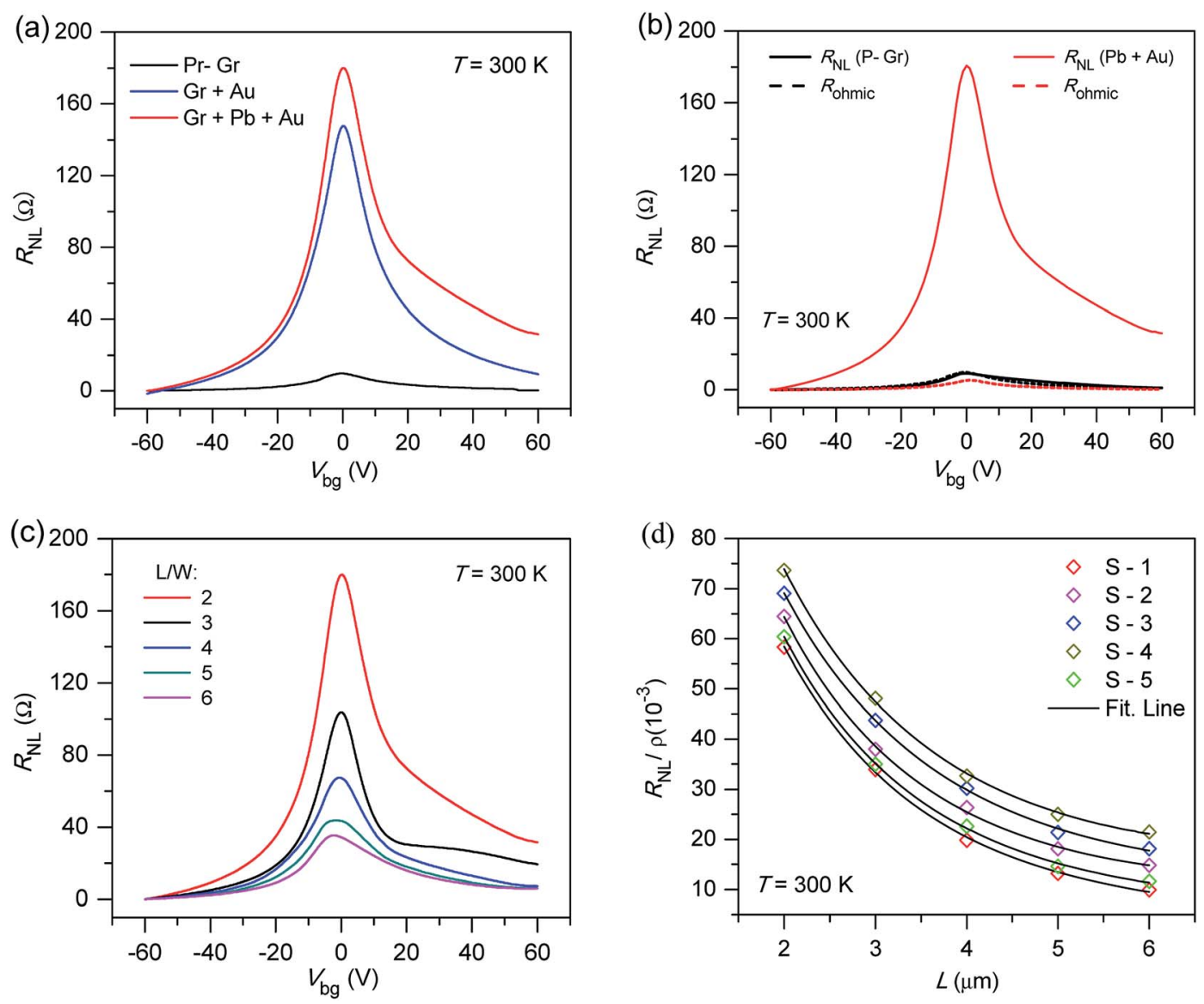

Fig. 2 (a) Comparison of non-local signal in pristine graphene, graphene $+\mathrm{Au}$, and graphene $+\mathrm{Pb}+\mathrm{Au}$. (b) Non-local resistance measurement of pristine graphene ( $\mathrm{P}-\mathrm{Gr}$ ) and under $\mathrm{Pb}$ device for $\mathrm{L} / \mathrm{W}=2$ as a function of back-gate voltages. Dotted lines are the ohmic contributions in $\mathrm{P}-\mathrm{Gr}$ and under $\mathrm{Pb}+\mathrm{Au}$. (c) Non-local measurement of the device under $\mathrm{Pb}+\mathrm{Au}$ (c) non-local resistance of graphene devices under $\mathrm{Pb}$ as a function of back-gate voltages at different $L / W$ ratios. (d) Non-local resistance near charge neutrality points of $\mathrm{Gr}$ as a function of a length under $\mathrm{Pb}+\mathrm{Au}$. We measured different samples as denoted by S-1, S-2, S-3, S-4, and S-5. 
Next, we turned our attention to confirming the origin of the large non-local (NL) signal we obtained; this was done by measuring the NL charge transport. We measured the length $(L)$ and width $(W)$ of the channel-dependent non-local signal to confirm its nature. We studied the length dependence of the non-local signal by keeping width fixed $(W=1 \mu \mathrm{m})$ and varying $L(2-6 \mu \mathrm{m})$. We measured the non-local signal of pristine $\mathrm{Gr}$ by applying a constant current $(I=0.99 \mu \mathrm{A})$ between two electrodes $(6 \& 2)$ and a detected a decrease in voltage $(V)$ across the neighbouring pair of electrodes ( $5 \& 3$ ). The NL signal obtained $\left(R_{\mathrm{NL}} \sim 9.6 \Omega\right)$ was not very high; it was also comparable in magnitude with the ohmic contribution shown in Fig. 2b. For ohmic measurement, we applied the current between the pair of electrodes $(1 \& 4)$ and measured the drop in $V$ between the other pair ( $2 \& 3$ ). The dimensions of the channel are $L=2 \mu \mathrm{m}$ and $W$ $=1 \mu \mathrm{m}$. The ohmic leakage contribution is calculated using the following:

$$
R_{\text {ohmic }}=\frac{4}{\pi} \rho \mathrm{e}^{(-\pi L / W)}
$$
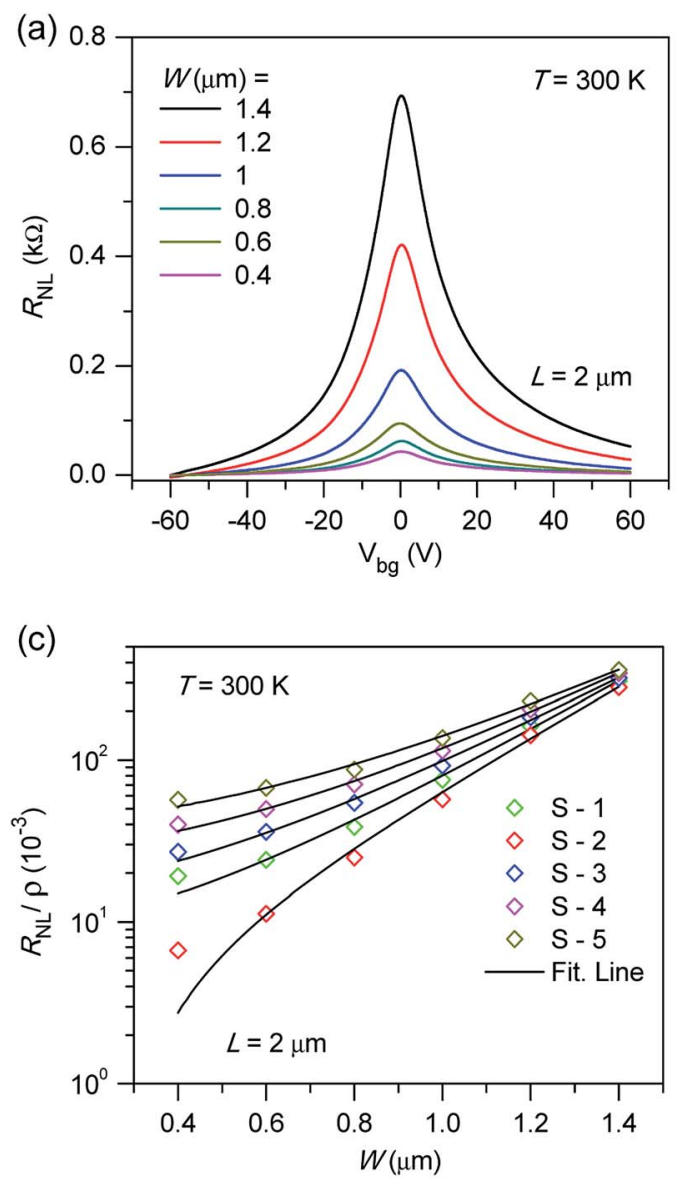

Thus there is no spin Hall effect (SHE) signature in pristine $\mathrm{Gr}^{13,31-33}$ therefore, it must have an extrinsic origin. To examine this, we deposited an Au layer on the Gr and measured an NL signal up to $R_{\mathrm{NL}}=150 \Omega$. Next, we used a thin layer of $\mathrm{Pb}$ as an intercalated layer between the $\mathrm{Gr}$ and Au. Following this, we measured the $\mathrm{Gr}$ under the $\mathrm{Pb}$ and $\mathrm{Au}$ in the non-local configuration. We observed a very large and strong non-local signal $\left(R_{\mathrm{NL}}=180 \Omega\right)$, and the contribution of ohmic leakage contribution is very small compared to pristine Gr, as shown in Fig. $2 \mathrm{~b}$. The value of $R_{\mathrm{NL}}$ we obtained is much higher than those previously reported. ${ }^{13,31}$

We have measured the non-local spin Hall signal of $\mathrm{Gr} / \mathrm{Pb} / \mathrm{Au}$ heterostructure by changing the thickness of $\mathrm{Pb}$ layer with a constant layer of $\mathrm{Au}(10 \mathrm{~nm})$. We used different thickness of intercalated $\mathrm{Pb}$ layer $(0 \mathrm{~nm}, 0.5 \mathrm{~nm}, 1 \mathrm{~nm}$, and $2 \mathrm{~nm})$. We confirmed the thickness of $\mathrm{Pb}$ and $\mathrm{Au}$ as shown in Fig. $4 \mathrm{a}$ and $\mathrm{b}$. We observed that the NL signal was increased with the increasing thickness of $\mathrm{Pb}$ layer (Fig. 4c). For the $\mathrm{Pb}$ layer thicker than $1 \mathrm{~nm}$, the magnitude of the NL signal saturates as shown in Fig. 4d. We attribute the large NL signal to the lattice
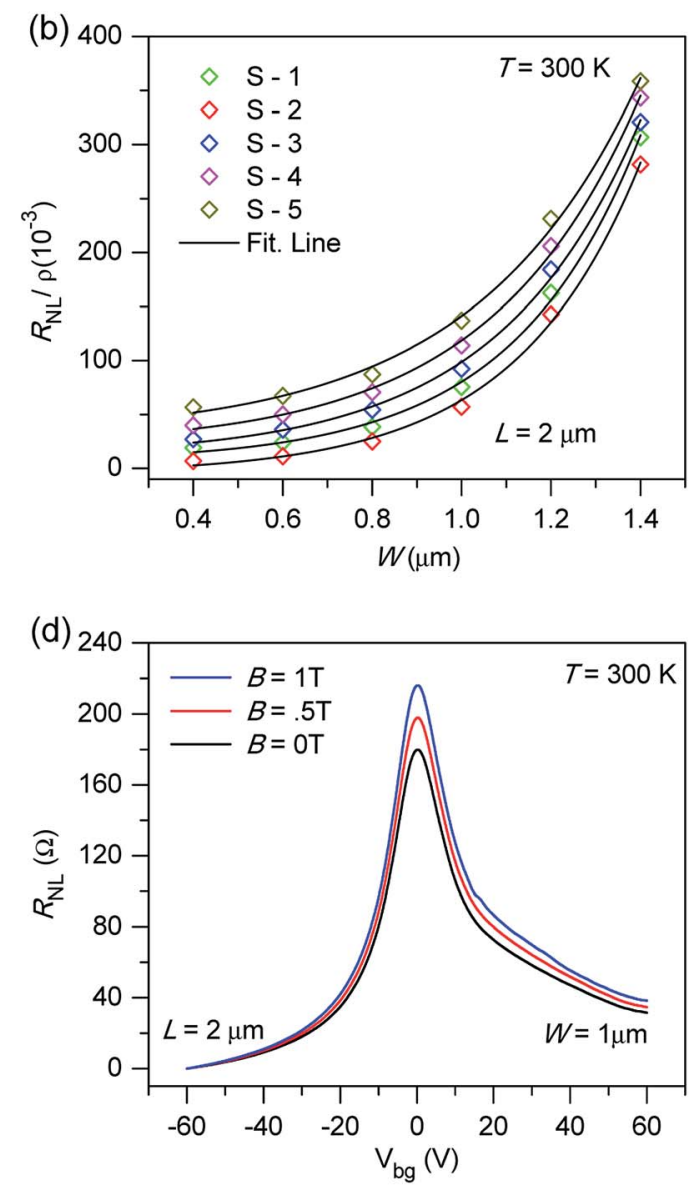

Fig. 3 (a) Non-local resistance of graphene devices under $\mathrm{Pb}+\mathrm{Au}$ as a function of back-gate voltages at different widths ( $W$ ) at a fixed length at 300 K. (b) Non-local resistance near charge neutrality points of $\mathrm{Gr}$ as a function of width under Pb in different samples denoted as S-1 to S-5. (c) Linear dependence of $\operatorname{Ln} R_{\mathrm{NL}}$ with $W$ that confirms that the signal comes from the SHE. We measured different samples denoted by S-1, S-2, S-3, S-4, and S-5. (d) Non-local resistance as a function of back-gate voltage at different perpendicular magnetic field room temperature with $L / W=$ $2 \mu \mathrm{m}$. This increment in the $R_{\mathrm{NL}}$ near the neutrality point (NP) can be assumed to be the combined effect of Zeeman splitting and bipolar transport at the charge NP under the perpendicular external magnetic field. 
mismatch among the graphene, $\mathrm{Pb}$, and $\mathrm{Au}$. It was previously reported that the intercalated $\mathrm{Pb}$ atoms were located in the equivalent positions to graphene $\mathrm{A}$ and $\mathrm{B}$ sublattices as compared to other heavy metal atoms. The intercalated layer of $\mathrm{Pb}$ significantly modified the band structure of graphene. ${ }^{34}$ Basically, the $\mathrm{Pb}$ atoms are more diffusive in the closer vicinity of the carbon atoms and enhance the SOI in graphene. At a larger thickness, the effect of $\mathrm{Pb}$ saturates due to filling of vicinity states of graphene. In addition, the $\mathrm{Pb}$ and $\mathrm{Gr}$ make a larger hybridization because the $\mathrm{Pb}$ layer is adjacent to the $\mathrm{Gr}$ sheet. The hybridization and charge carrier transfer to Gr sheet strongly affect electronic properties of graphene and SOI in graphene. $^{35}$

When $\mathrm{Pb}$ was used as an intercalated layer between graphene and $\mathrm{Au}$, the electronic structure of $\mathrm{Gr}$ was improved and electrons maintained a strong SOI during tunneling through $\mathrm{Pb} .^{34-36}$ Further, we estimated the NL signals as a function of the backgate voltage at different values of $L(2-5 \mu \mathrm{m})$ as shown in Fig. 2c. We noted that the NL signal decreased as the length of the device decreased. To determine key spin parameters such as the spin diffusion length $\left(\lambda_{\mathrm{s}}\right)$, spin relaxation time $\left(\tau_{\mathrm{s}}\right)$, and SOI in our heterostructure device, we performed length-dependent NL signal measurements. We plotted $R_{\mathrm{NL}} / \rho$ against the length of the channel and the resultant cures were fitted by the spin diffusion equation, ${ }^{32,37}$

$$
R_{\mathrm{NL}}=\frac{1}{2} \gamma^{2} \rho \frac{W}{\lambda_{\mathrm{s}}} \mathrm{e}^{-L / \lambda_{\mathrm{S}}}=\frac{1}{2} \gamma^{2} \rho \frac{W^{2}}{\lambda_{\mathrm{so}}{ }^{2}} \mathrm{e}^{-L W / \lambda_{\mathrm{so}}{ }^{2}}
$$

where $\lambda_{\mathrm{s}}$ is the spin diffusion length and $\gamma$ is the spin Hall coefficient. $^{32} \lambda_{\text {so }}$ is the spin precession length, which is related to the spin relaxation length as $\lambda_{\mathrm{s}}=\frac{\lambda_{\mathrm{so}}{ }^{2}}{W} \cdot{ }^{38}$ We extract $\lambda_{\mathrm{s}}$ and $\gamma$ in the range of $(0.20-0.62 \mu \mathrm{m})$ and $(0.170-0.32)$ near the CNP, respectively.

Further, confirming the nature of the SHE, we performed width-dependent NL signal measurements. Fig. 3a shows the NL signal as a function of the back-gate voltage with a fixed channel length $(L=1 \mu \mathrm{m})$ and varying channel width $(W=0.4-$ $1.4 \mu \mathrm{m})$. In this case, when varying the width, we only selected the devices in which the condition $\lambda_{s}, \lambda_{\text {so }}>W$ was fulfilled. The NL signal increased as the width of channel increased. Then, we plotted $R_{\mathrm{NL}} / \rho$ as a function of $W$ near the CNP again and fitted the data by using the spin diffusion equation as shown in Fig. $3 \mathrm{~b}$. Thus, the estimated values of $\lambda_{\mathrm{s}}$ and $\gamma$ were in the range of $(0.120-0.72 \mu \mathrm{m})$ and $(0.12-0.36)$ and showed good agreement with the length-dependent NL signals. Fig. 3c shows the linear (a)
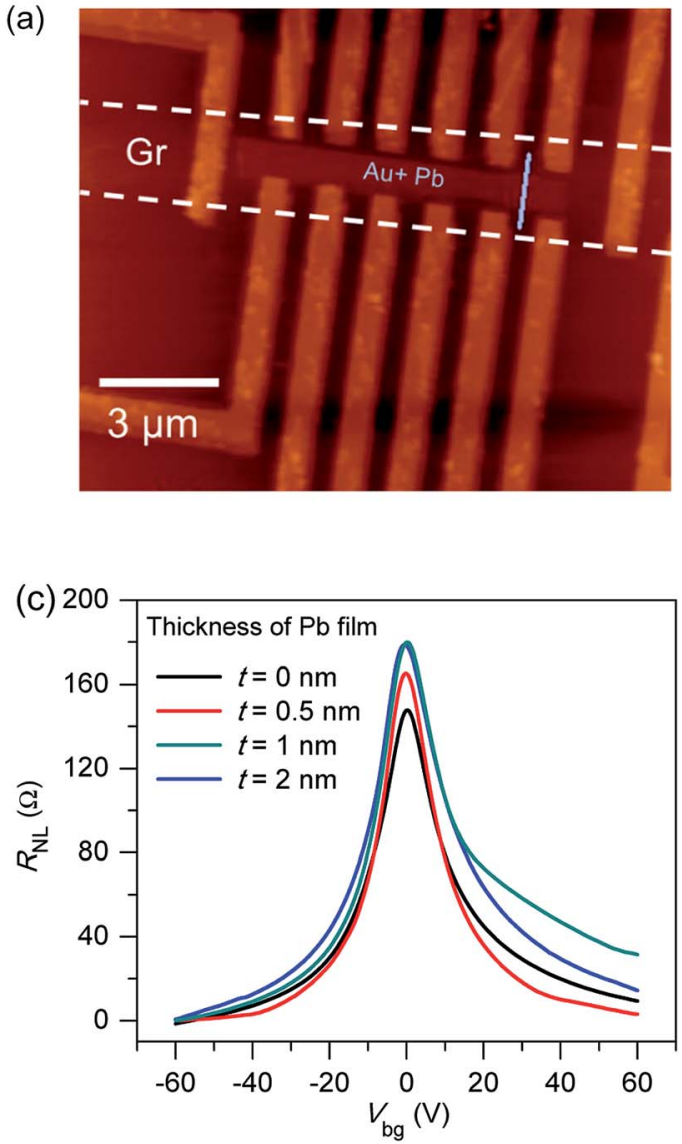
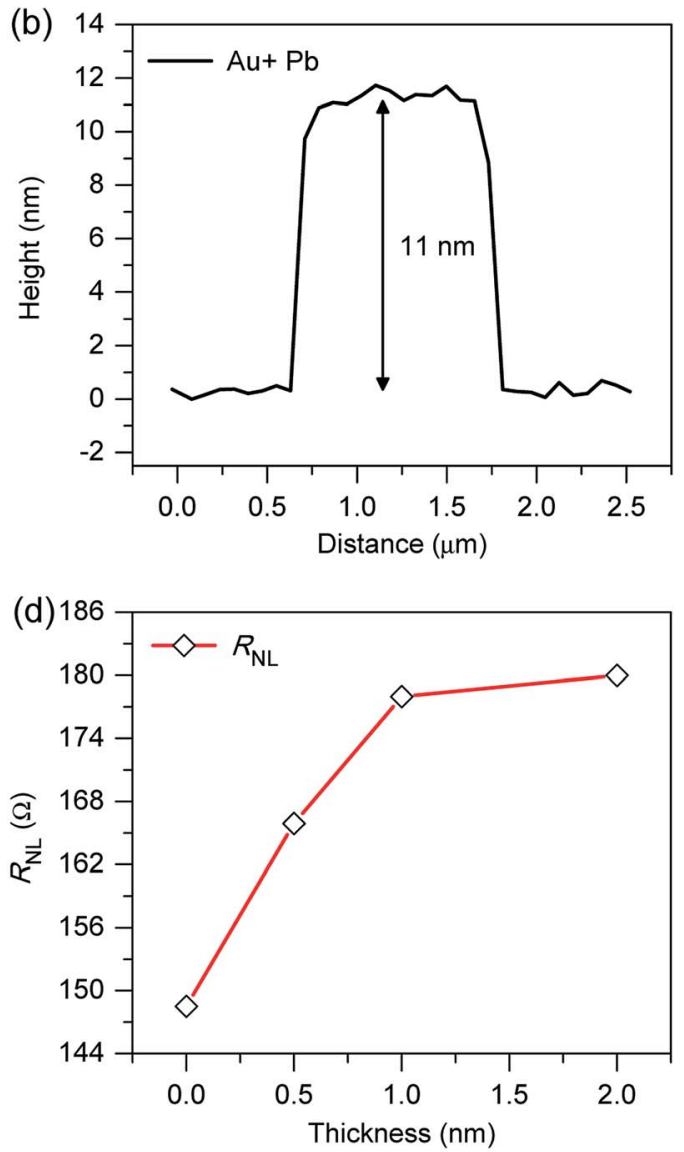

Fig. 4 (a) Atomic force microscopy image of the final device to confirm the thickness of $\mathrm{Au}$ and $\mathrm{Pb}$ (b) height profile of $\mathrm{Au}$ and $\mathrm{Pb}$ region. Total height is $11 \mathrm{~nm}$ which includes $1 \mathrm{~nm} \mathrm{~Pb}$ and $10 \mathrm{~nm} \mathrm{Au}$. (c) Non-local signal with respect to the thickness of Pb layer. (d) Change in non-local signal with respect to thickness of the $\mathrm{Pb}$ film. 
dependence of $\ln \left(R_{\mathrm{NL}} / \rho\right)$ on $W$, which also confirms that the SHE shows a power-law dependence. The linear dependency of NL signals confirmed that the expected signal is caused by the SHE. If the dominant NL signal is caused owing to ohmic contribution, non-linear behaviour should be observed. ${ }^{31}$ Notably, we observed a giant and obvious NL signal as compared to ohmic contribution. Such a signal can only be observed when the width of the channel is smaller than the distance between the electrode. ${ }^{32,37}$ Fig. 3d shows NL resistance as a function of the back-gate voltage $\left(V_{\mathrm{bg}}\right)$ at different perpendicular magnetic fields $\left(B_{\perp}\right)$ at $300 \mathrm{~K}$ with $L / W=2$. This increment in $R_{\mathrm{NL}}$ and Hall resistivity near the CNP can be attributed to the combined effect of Zeeman splitting and bipolar transport at the CNP under an external magnetic field. ${ }^{39,40}$

Moreover, to confirm the origin of the observed NL signal, we measured the current-voltage $(I-V)$ characteristics. The linear relationship between NL voltage and the applied current excludes any possible contribution of the thermoelectric effect as shown in Fig. S2.† We can exclude such a contribution because we measured the NL voltage drop along the width of the channel and the temperature gradient due to the thermoelectric effect acts along the length of the sample. We also measured the resistance as a function of the temperature as shown in Fig. S3. $\dagger$ We observed a logarithmic increase in resistance with temperature at the CNP. This effect may originate from WL, disorderness due to electron-electron interaction, or the Kondo effect. ${ }^{\mathbf{4 1 , 4 2}}$

We evaluated $\tau_{\mathrm{s}}$ by $\tau_{\mathrm{s}}=\lambda_{\mathrm{s}}{ }^{2} / D$, where $D$ is the diffusion constant and can be obtained using the Einstein relation $\sigma=$ $e^{2} D_{\mathrm{c}} N_{2 \mathrm{D}}\left(E_{\mathrm{F}}\right)$. Here, $N_{2 \mathrm{D}}\left(E_{\mathrm{F}}\right)$ is the density of the state of Gr. Thus, the estimated value of the spin relaxation time was $\tau_{\mathrm{s}} \sim 2-4 \mathrm{ps}$. We also estimated the strength of the SOI in our device. For this estimation, we assumed that the Elliott-Yafet dephasing mechanism is dominant in our case because the spin relaxation time is directly proportional to the momentum scattering time $\left(\tau_{\mathrm{s}} \propto \tau_{\mathrm{p}}\right)^{31,43,44}$ as shown in Fig. 6a. We calculated the SOI using the following relation, $\Delta_{\text {so }}=\varepsilon_{\mathrm{F}} \sqrt{\frac{\tau_{\mathrm{p}}}{\tau_{\mathrm{S}}}}$, where $\varepsilon_{\mathrm{F}}$ is the Fermi energy and $\tau_{\mathrm{p}}$ is the momentum scattering time $\left(\tau_{\mathrm{p}}=\frac{\hbar \sqrt{\pi / n}}{\rho e^{2} v_{\mathrm{F}}}\right)$, and $v_{\mathrm{F}}$ is the Fermi velocity. The obtained value of SOI is 70-90 meV, which is greater than previously reported values in hydrogenated graphene $(\approx 2.5 \mathrm{meV})$ and fluorinated graphene $(\approx 5.1 \mathrm{meV}) .{ }^{12,13}$ Thus, the presence and enhancement in the NL signal at room temperature and zero magnetic field $(B=0 \mathrm{~T})$ enhances the SOI in Gr. ${ }^{2,30,45}$ The spin current in Gr via the SHE and the inverse SHE could be detected as the SOI was enhanced.
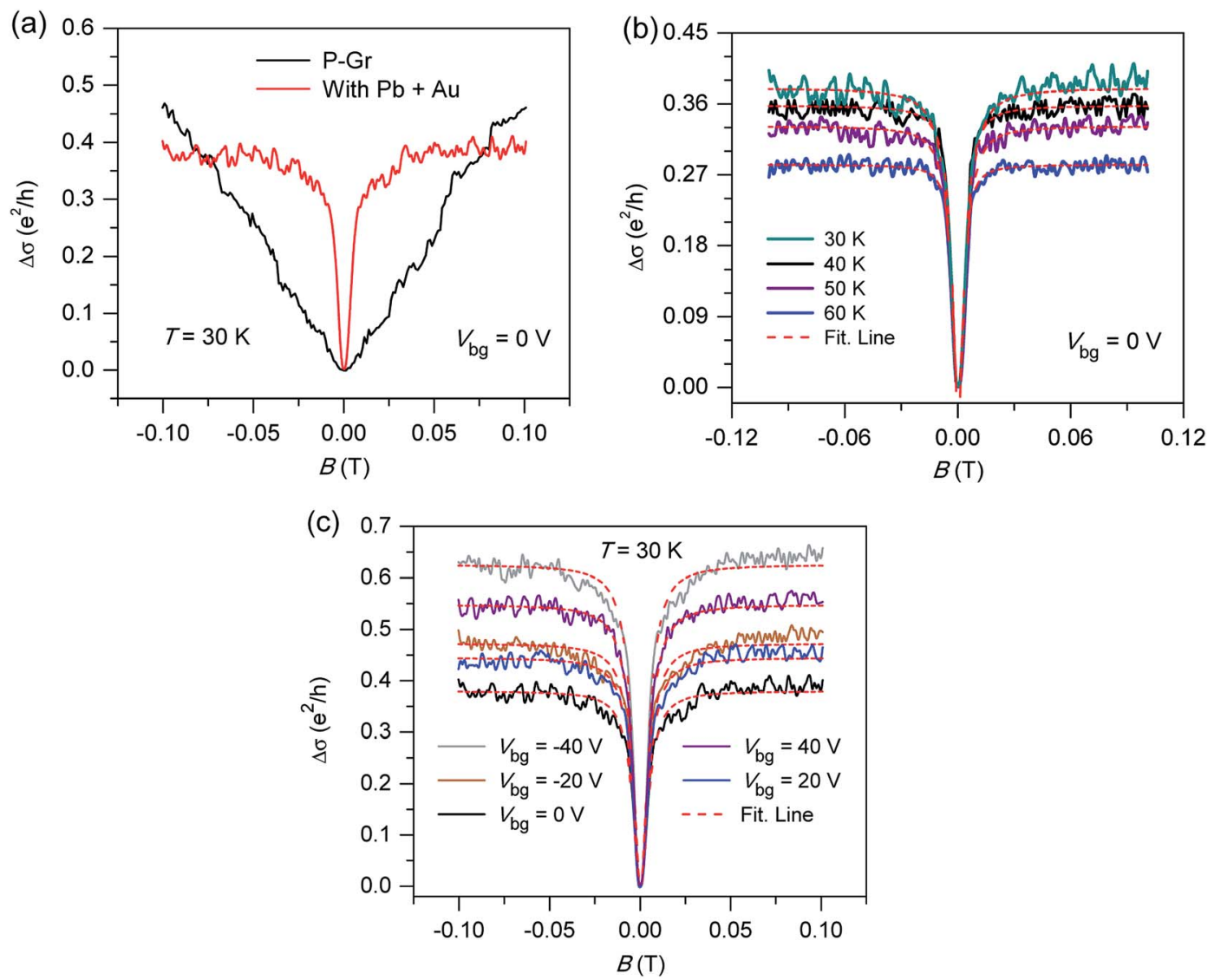

Fig. 5 (a) Magnetoconductance (MC) curves measured before and after the deposition of $\mathrm{Pb}+\mathrm{Au}$ at $30 \mathrm{~K}$ (b) weak localization curves at various temperatures; the red line is the fitting line. (c) Back gate-dependent weak localization curves at $30 \mathrm{~K}$. 
We further estimated the strength of the SOI in the Gr sheet by analyzing the WL signal near the zero field. ${ }^{46,47}$ The WL signal was dominant near $B=0 \mathrm{~T}$ as compared to pristine $\mathrm{Gr}$ as shown in Fig. 5a. We could observe the WL signals only at low temperatures as shown in Fig. 5b. We also measured the gate dependent magnetoconductance at $30 \mathrm{~K}$, as shown in Fig. 5c. The suppression of the WL signal agreed with the Kane-Mele SOI in our measurement. Thus, we used the following equation to fit the WL data, ${ }^{46}$

$$
\begin{aligned}
\Delta \sigma(B)= & -\frac{e^{2}}{2 \pi h}\left[-F\left(\frac{B}{B_{\varphi}+B_{\mathrm{i}}}\right)-2 F\left(\frac{B}{B_{\varphi}+B_{\mathrm{so}}}\right)\right. \\
& +4 F\left(\frac{B}{B_{\varphi}+B_{*}+B_{\mathrm{so}}}\right)+2 F\left(\frac{B}{B_{\varphi}+2 B_{\mathrm{i}}+B_{\mathrm{so}}}\right) \\
& \left.+F\left(\frac{B}{B_{\varphi}+2 B_{*}}\right)\right]
\end{aligned}
$$

Here, $F(x)=\ln (x)+\Psi(1 / 2+1 / x)$, where $\Psi(x)$ is the digamma function, $B_{\varphi, \mathrm{i},{ }^{*}, \mathrm{so}}=\left(\frac{\hbar c}{4 D e}\right) \tau_{\varphi, \mathrm{i},{ }^{*}, \mathrm{so}}{ }^{-1}, D$ is the charge carrier diffusion constant, and $\tau_{\varphi}^{-1}$ is the dephasing rate. $\tau_{\mathrm{i}}^{-1}$ describes the inter-valley scattering time, $\tau_{*}{ }^{-1}$ is the intra-valley scattering time, and $\tau_{\text {so }}{ }^{-1}$ is the intrinsic KM spin relaxation time. Using the temperature-dependent WL data, we extracted the dephasing rate as shown in Fig. 6b. The dephasing length decreased as the temperature increased from 30 to $60 \mathrm{~K}$, which can be attributed to electron-electron interaction. Moreover, $\tau_{\mathrm{so}}$ can be related to the strength of the SOI by $\Delta_{\text {so }}=\varepsilon_{\mathrm{F}} \sqrt{\frac{\tau_{\mathrm{p}}}{\tau_{\mathrm{s}}}}$, where $\varepsilon_{\mathrm{F}}$ is the Fermi energy. The estimated value of the SOI is 50-60 $\mathrm{meV}$. The temperature- and gate-dependent SOIs are shown in Fig. $6 \mathrm{c}$ and $\mathrm{d}$. The SOI was observed to vary with temperature; this change may be owing to the contribution of temperaturedependent scattering, ionic centers, and phonons.

In summary, we fabricated Gr FET, in which $\mathrm{Pb}$ was used as the intercalated layer between $\mathrm{Gr}$ and $\mathrm{Au}$. We measured the electronic properties in pristine $\mathrm{Gr}$ and $\mathrm{Gr}$ with $\mathrm{Pb}+\mathrm{Au}$; their mobilities were $25000 \mathrm{~cm}^{2} \mathrm{~V}^{-1} \mathrm{~s}^{-1}$ and $29100 \mathrm{~cm}^{2} \mathrm{~V}^{-1} \mathrm{~s}^{-1}$, respectively. The mobility increased by the intercalation of $\mathrm{Pb}$ because it modified the electronic structure of Gr. To confirm the SHE, we performed length- and width-dependent NL signal measurements in pristine $\mathrm{Gr}$ and with adatoms. We observed a giant NL signals of $150 \Omega$ and $180 \Omega$ with only $\mathrm{Au}$ and with $\mathrm{Pb}+$ $\mathrm{Au}$, respectively. Further, we calculated key parameters such as spin $\lambda_{\mathrm{s}}, \tau_{\mathrm{s}}$, and $\gamma$ in the range of $(0.20-0.62 \mu \mathrm{m}),(0.40-0.62 \mathrm{ps})$, and (0.170-0.32), respectively, near the CNP. The strength of the SOI was 80-90 meV. Moreover, we performed the WL
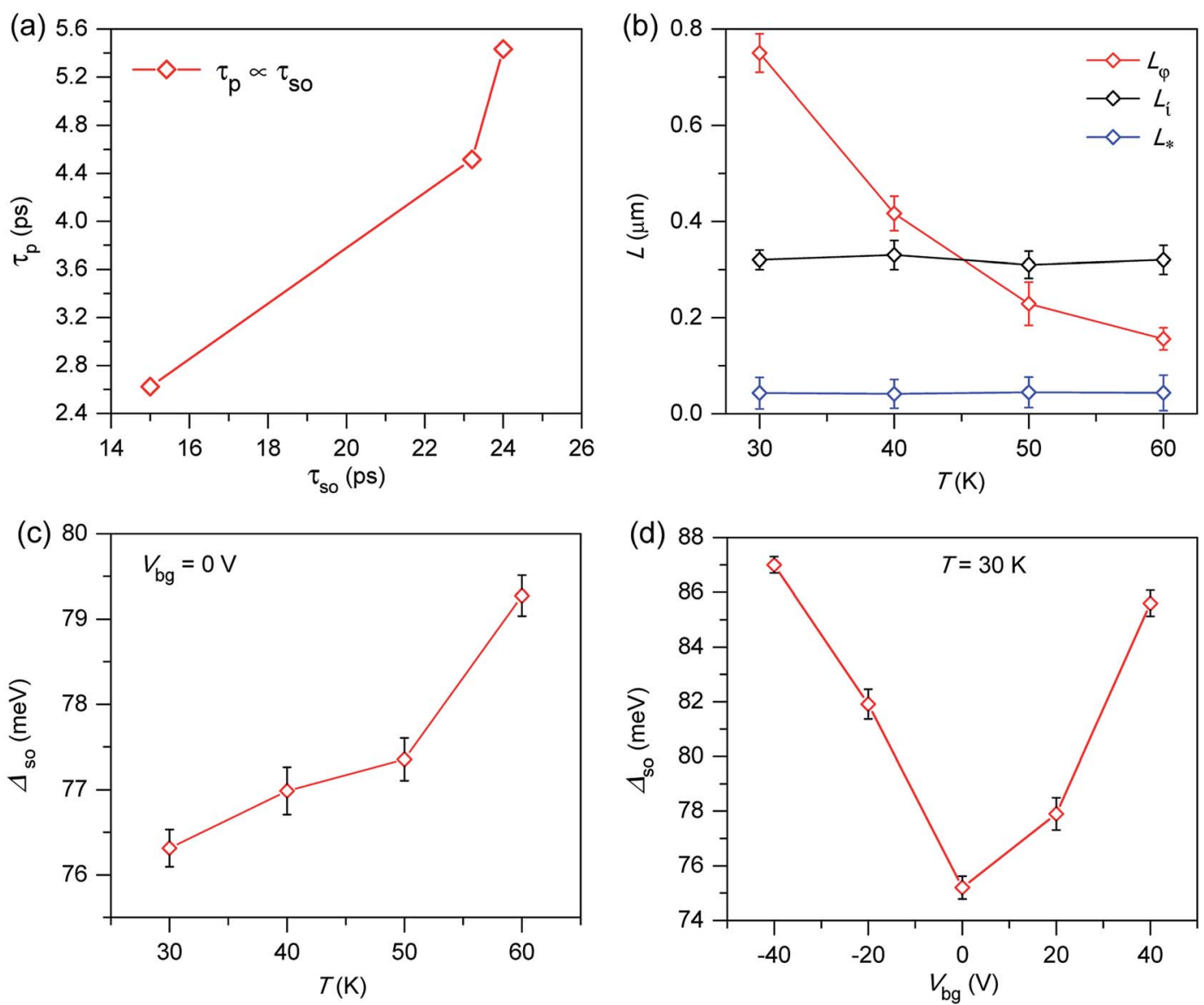

Fig. 6 (a) Relation between spin relaxation time and momentum scattering time (b) temperature-dependent dephasing length $L_{\varphi}$ with error bars derived from WL formula fitting. (c) Temperature-dependent spin-orbital interaction of graphene under $\mathrm{Pb}$ and $\mathrm{Au}$ that linearly increases with temperature. (d) Gate-dependent SOI in graphene under Pb and Au. 
measurements to confirm the self-consistency. The SOI strength was close to the calculated value according to the NL signal measurements. The thin metal films on Gr lead to an enhanced SOI and a SHE with a large spin Hall coefficient. The SHE and SOI were enhanced by the proximity-induced effect due to adatoms of heavy metals. ${ }^{34,45}$ In particular, the observed SHE stems from extrinsic origins such as skew scattering and quantum-side jumps.

\section{Device fabrication}

A Gr flake from commercial graphite was exfoliated micromechanically on $\mathrm{SiO}_{2}$ /highly p-doped $\mathrm{Si}(300 \mathrm{~nm})$ substrate by using the standard Scotch tape method. A monolayer Gr can be identified with appropriate thickness due to the interference effect. Further, Raman spectroscopy was used to confirm the thickness of Gr. After making the large pattern, the samples were annealed at $400{ }^{\circ} \mathrm{C}$ for $6 \mathrm{~h}$ under $\mathrm{Ar} / \mathrm{H}_{2}\left(97.5 \% \mathrm{Ar} / 2.5 \% \mathrm{H}_{2}\right)$ gas flow. Electron-beam lithography (EBL) and $\mathrm{O}_{2}$ plasma were used to design the electrodes in the Hall bar $(H)$ configuration, and $\mathrm{Cr} / \mathrm{Au}(6 / 60 \mathrm{~nm})$ was deposited by thermal evaporation. Moreover, we made a small window with EBL and deposited $\mathrm{Pb}$ and $\mathrm{Au}(1 \mathrm{~nm} / 10 \mathrm{~nm})$ thin films through thermal evaporation. The length and width of the Gr channel were 1-6 $\mu \mathrm{m}$ and 0.4$1.4 \mu \mathrm{m}$, respectively.

The Raman spectra of Gr were measured with a Renishaw micro-spectrometer over a wavenumber range of 1100$3200 \mathrm{~cm}^{-1}$, with a laser wavelength of $514.5 \mathrm{~nm}$. To prevent the device from experiencing local heating, the spot size was fixed as $1 \mu \mathrm{m}$, and the power was maintained at $1.0 \mathrm{~mW}$. To examine the surface morphology of $\mathrm{Gr}$, an atomic force microscope (AFM) was used. The local and non-local measurements of the $\mathrm{Gr} / \mathrm{Pb} / \mathrm{Au}$ heterostructure device were performed by using the standard lock-in technique at different temperatures with or without a magnetic field.

\section{Conflicts of interest}

There are no conflicts to declare.

\section{Acknowledgements}

This work was supported by the Basic Science Research Program (2016R1D1A1A09917762, 2019R1F1A1057697) and the Global Research and Development Center Program (2018K1A4A3A01064272) through the National Research Foundation of Korea (NRF) grant funded by the Korea government (Ministry of Education and the Ministry of Science and ICT).

\section{References}

1 S. A. Wolf, D. D. Awschalom, R. A. Buhrman, J. M. Daughton, S. von Molnár, M. L. Roukes, A. Y. Chtchelkanova and D. M. Treger, Science, 2001, 294, 1488-1495.

2 K. S. Novoselov, A. K. Geim, S. V. Morozov, D. Jiang, Y. Zhang, S. V. Dubonos, I. V. Grigorieva and A. A. Firsov, Science, 2004, 306, 666-669.
3 A. K. Geim and K. S. Novoselov, in Nanoscience and Technology: A Collection of Reviews from Nature Journals, World Scientific, 2010, pp. 11-19.

4 N. Tombros, C. Jozsa, M. Popinciuc, H. T. Jonkman and B. J. Van Wees, Nature, 2007, 448, 571.

5 M. V. Kamalakar, C. Groenveld, A. Dankert and S. P. Dash, Nat. Commun., 2015, 6, 6766.

6 W. Han, R. K. Kawakami, M. Gmitra and J. Fabian, Nat. Nanotechnol., 2014, 9, 794.

7 S. Datta and B. Das, Appl. Phys. Lett., 1990, 56, 665-667.

8 A. H. Castro Neto and F. Guinea, Phys. Rev. Lett., 2009, 103, 026804.

9 F. Schedin, A. K. Geim, S. V. Morozov, E. W. Hill, P. Blake, M. I. Katsnelson and K. S. Novoselov, Nat. Mater., 2007, 6, 652.

10 F. Kuemmeth, S. Ilani, D. Ralph and P. McEuen, Nature, 2008, 452, 448.

11 D. Huertas-Hernando, F. Guinea and A. Brataas, Phys. Rev. B: Condens. Matter Mater. Phys., 2006, 74, 155426.

12 J. Balakrishnan, G. K. W. Koon, M. Jaiswal, A. C. Neto and B. Özyilmaz, Nat. Phys., 2013, 9, 284.

13 A. Avsar, J. H. Lee, G. K. W. Koon and B. Özyilmaz, 2D Materials, 2015, 2, 044009.

14 H. Zhang, C. Lazo, S. Blügel, S. Heinze and Y. Mokrousov, Phys. Rev. Lett., 2012, 108, 056802.

15 C. Weeks, J. Hu, J. Alicea, M. Franz and R. Wu, Phys. Rev. X, 2011, 1, 021001.

16 M. J. Piotrowski, P. Piquini and J. L. Da Silva, Phys. Rev. B: Condens. Matter Mater. Phys., 2010, 81, 155446.

17 S. Blügel, Phys. Rev. Lett., 1992, 68, 851-854.

18 S. V. Morozov, K. S. Novoselov, M. I. Katsnelson, F. Schedin, L. A. Ponomarenko, D. Jiang and A. K. Geim, Phys. Rev. Lett., 2006, 97, 016801.

19 H. Haugen, D. Huertas-Hernando and A. Brataas, Phys. Rev. B: Condens. Matter Mater. Phys., 2008, 77, 115406.

20 P. Wei, S. Lee, F. Lemaitre, L. Pinel, D. Cutaia, W. Cha, F. Katmis, Y. Zhu, D. Heiman and J. Hone, Nat. Mater., 2016, 15, 711.

21 Z. Wang, C. Tang, R. Sachs, Y. Barlas and J. Shi, Phys. Rev. Lett., 2015, 114, 016603.

22 Z. Wang, D. K. Ki, H. Chen, H. Berger, A. H. MacDonald and A. F. Morpurgo, Nat. Commun., 2015, 6, 8339.

23 A. Avsar, J. Y. Tan, T. Taychatanapat, J. Balakrishnan, G. K. W. Koon, Y. Yeo, J. Lahiri, A. Carvalho, A. S. Rodin, E. C. T. O'Farrell, G. Eda, A. H. Castro Neto and B. Özyilmaz, Nat. Commun., 2014, 5, 4875.

24 A. M. Afzal, M. F. Khan, G. Nazir, G. Dastgeer, S. Aftab, I. Akhtar, Y. Seo and J. Eom, Sci. Rep., 2018, 8, 3412.

25 A. C. Ferrari, J. C. Meyer, V. Scardaci, C. Casiraghi, M. Lazzeri, F. Mauri, S. Piscanec, D. Jiang, K. S. Novoselov, S. Roth and A. K. Geim, Phys. Rev. Lett., 2006, 97, 187401.

26 D. Graf, F. Molitor, K. Ensslin, C. Stampfer, A. Jungen, C. Hierold and L. Wirtz, Nano Lett., 2007, 7, 238-242.

27 J. Y. Tan, A. Avsar, J. Balakrishnan, G. K. W. Koon, T. Taychatanapat, E. C. T. O'Farrell, K. Watanabe, T. Taniguchi, G. Eda, A. H. C. Neto and B. Özyilmaz, Appl. Phys. Lett., 2014, 104, 183504. 
28 Z. Wang, D.-K. Ki, J. Y. Khoo, D. Mauro, H. Berger, L. S. Levitov and A. F. Morpurgo, Phys. Rev. X, 2016, 6, 041020.

29 F. V. Tikhonenko, D. W. Horsell, R. V. Gorbachev and A. K. Savchenko, Phys. Rev. Lett., 2008, 100, 056802.

30 K. Kim, S. Larentis, B. Fallahazad, K. Lee, J. Xue, D. C. Dillen, C. M. Corbet and E. Tutuc, ACS Nano, 2015, 9, 4527-4532.

31 J. Balakrishnan, G. Kok Wai Koon, M. Jaiswal, A. H. Castro Neto and B. Özyilmaz, Nat. Phys., 2013, 9, 284.

32 D. Abanin, A. Shytov, L. Levitov and B. Halperin, Phys. Rev. B: Condens. Matter Mater. Phys., 2009, 79, 035304.

33 G. Mihajlović, J. Pearson, M. Garcia, S. Bader and A. Hoffmann, Phys. Rev. Lett., 2009, 103, 166601.

34 F. Calleja, H. Ochoa, M. Garnica, S. Barja, J. J. Navarro, A. Black, M. M. Otrokov, E. V. Chulkov, A. Arnau and A. L. V. De Parga, Nat. Phys., 2015, 11, 43.

35 I. I. Klimovskikh, M. M. Otrokov, V. Y. Voroshnin, D. Sostina, L. Petaccia, G. Di Santo, S. Thakur, E. V. Chulkov and A. M. Shikin, ACS Nano, 2017, 11, 368-374.

36 D. Ma and Z. Yang, New J. Phys., 2011, 13, 123018. 37 S. Kettemann, Phys. Rev. Lett., 2007, 98, 176808.

38 J. Balakrishnan, G. K. W. Koon, A. Avsar, Y. Ho, J. H. Lee, M. Jaiswal, S.-J. Baeck, J.-H. Ahn, A. Ferreira and M. A. Cazalilla, Nat. Commun., 2014, 5, 4748.
39 D. Abanin, R. Gorbachev, K. Novoselov, A. Geim and L. Levitov, Phys. Rev. Lett., 2011, 107, 096601.

40 D. Abanin, S. Morozov, L. Ponomarenko, R. Gorbachev,

A. Mayorov, M. Katsnelson, K. Watanabe, T. Taniguchi, K. Novoselov and L. Levitov, Science, 2011, 332, 328-330.

41 S. Lara-Avila, A. Tzalenchuk, S. Kubatkin, R. Yakimova, T. Janssen, K. Cedergren, T. Bergsten and V. Fal'ko, Phys. Rev. Lett., 2011, 107, 166602.

42 J.-H. Chen, L. Li, W. G. Cullen, E. D. Williams and M. S. Fuhrer, Nat. Phys., 2011, 7, 535.

43 H. Ochoa, A. C. Neto and F. Guinea, Phys. Rev. Lett., 2012, 108, 206808.

44 D. Huertas-Hernando, F. Guinea and A. Brataas, Phys. Rev. Lett., 2009, 103, 146801.

45 J. Balakrishnan, G. K. W. Koon, A. Avsar, Y. Ho, J. H. Lee, M. Jaiswal, S.-J. Baeck, J.-H. Ahn, A. Ferreira, M. A. Cazalilla, A. H. C. Neto and B. Özyilmaz, Nat. Commun., 2014, 5, 4748.

46 E. McCann and V. I. Fal'ko, Phys. Rev. Lett., 2012, 108, 166606.

47 E. McCann and M. Koshino, Phys. Rev. B: Condens. Matter Mater. Phys., 2010, 81, 241409. 